\title{
Diagnostic Value of Systemic Inflammatory Response Index for Catheter-Related Bloodstream Infection in Patients Undergoing Haemodialysis
}

\author{
Jiajia Yang $\mathbb{D},{ }^{1}$ Hongmei Wang, ${ }^{1}$ Qing Hua, ${ }^{2}$ Jian $W u\left(\mathbb{D},{ }^{3}\right.$ and Ying Wang $\mathbb{D}^{1}$ \\ ${ }^{1}$ Department of Infection Management, The Affiliated Suzhou Hospital of Nanjing Medical University, Suzhou Municipal Hospital, \\ Gusu School, Nanjing Medical University, Suzhou, China \\ ${ }^{2}$ Department of Nephrology, The Affiliated Suzhou Hospital of Nanjing Medical University, Suzhou Municipal Hospital, \\ Gusu School, Nanjing Medical University, Suzhou, China \\ ${ }^{3}$ Department of Clinical Laboratory, The Affiliated Suzhou Hospital of Nanjing Medical University, Suzhou Municipal Hospital, \\ Gusu School, Nanjing Medical University, Suzhou, China
}

Correspondence should be addressed to Ying Wang; wangy830224@sina.com

Received 30 November 2021; Revised 31 December 2021; Accepted 5 January 2022; Published 29 January 2022

Academic Editor: Zhen-Jian Zhuo

Copyright (c) 2022 Jiajia Yang et al. This is an open access article distributed under the Creative Commons Attribution License, which permits unrestricted use, distribution, and reproduction in any medium, provided the original work is properly cited.

\begin{abstract}
Objective. This study was aimed at investigating the diagnostic value of the neutrophil-to-lymphocyte ratio (NLR), platelet-tolymphocyte ratio (PLR), C-reactive protein-to-albumin ratio (CAR), and systemic inflammatory response index (SIRI) for catheter-related bloodstream infection (CRBSI) in patients undergoing haemodialysis. Methods. A total of 296 patients undergoing haemodialysis with vascular access were selected and divided into the infected (58 patients) and uninfected (238 patients) groups. Their aetiological and general characteristics were retrospectively collected. The NLR, PLR, CAR, and SIRI were calculated. Results. The NLR, PLR, CAR, and SIRI values in the infected group were significantly higher than those in the uninfected group $(P<0.05)$. After the anti-infective treatment, the NLR, PLR, CAR, and SIRI values in patients with CRBSI were significantly decreased $(P<0.05)$. The NLR, CAR, and SIRI showed diagnostic efficacy in patients with CRBSI with cutoff values of 4.485 (area under the curve $(\mathrm{AUC})=0.827,95 \%$ confidence interval $(\mathrm{CI})=0.768-0.887), 0.975(\mathrm{AUC}=0.836,95 \%$ $\mathrm{CI}=0.779-0.892)$, and $3.390(\mathrm{AUC}=0.947,95 \% \mathrm{CI}=0.919-0.976)$. The CAR and SIRI values in patients with gram-negative bacterial infection were significantly higher than those with gram-positive bacterial infection $(P<0.05)$. The AUCs of CAR and SIRI were $0.693(0.537-0.848)$ and $0.821(0.700-0.942)$ in differentiating gram-negative and gram-positive bacterial infections, respectively. Conclusion. Our results showed SIRI as a novel and efficient indicator for the early diagnosis of CRBSI in patients undergoing haemodialysis.
\end{abstract}

\section{Introduction}

Vascular access is indispensable in haemodialysis [1], with the central venous catheter (CVC) being the most common owing to the following advantages: easy operation, plug and play, high blood flow, and minimal damage. However, as it is an invasive procedure, complications such as deep vein thrombosis, venous stenosis, and catheter-related bloodstream infection (CRBSI) can also occur [2]. Moreover, if CRBSI cannot be timely controlled, some serious complications, such as bacteraemia, brain abscess, endocarditis, and even septic shock, may occur, which greatly increase the patients' mortality [3]. Therefore, early diagnosis and treatment of CRBSI are crucial to improve the prognosis of patients undergoing haemodialysis via the CVC.

Currently, blood culture is the gold standard for the diagnosis of bloodstream infections [4]. However, owing to the long culture cycle and low positive rate of blood culture, patients may miss the best opportunity for diagnosis and treatment. Therefore, if simple, low-cost, and less-invasive routine detection methods such as blood routine can be established, indicators that can be used for the early 
TABLE 1: Distribution characteristics of CRBSI pathogens.

\begin{tabular}{lcc}
\hline Pathogen type & Number & Proportion (\%) \\
\hline Gram-negative bacteria $\left(\mathrm{G}^{-}\right)$ & 40 & \\
Escherichia coli & 19 & 47.50 \\
Pseudomonas aeruginosa & 10 & 25.00 \\
Stenotrophomonas maltophilia & 3 & 7.50 \\
Klebsiella pneumoniae & 2 & 5.00 \\
Acinetobacter baumannii & 2 & 5.00 \\
Acinetobacter jonesi & 1 & 2.50 \\
Others & 3 & 7.50 \\
Gram positive bacteria $\left(\mathrm{G}^{+}\right)$ & 23 & \\
Staphylococcus aureus & 9 & 39.13 \\
Staphylococcus capitis & 5 & 21.73 \\
Excrement enterococcus & 4 & 17.39 \\
Staphylococcus epidermidis & 2 & 8.70 \\
Dung enterococcus & 2 & 8.70 \\
Staphylococcus haemolyticus & 1 & 4.35 \\
Fungus & 4 & \\
Candida albicans & 2 & 50.00 \\
Aspergillus & 2 & 50.00 \\
\hline
\end{tabular}

diagnosis of CRBSI should be determined, which can help prevent and treat CRBSI in patients undergoing haemodialysis, to improve the prognosis. Previous studies have shown that neutrophil-to-lymphocyte ratio (NLR), platelet-tolymphocyte ratio (PLR), and C-reactive protein-to-albumin ratio (CAR) have certain values for the early diagnosis of nosocomial infection [5-7]. Nevertheless, only a few reports have demonstrated the significance of these indicators in the early diagnosis of CRBSI in patients undergoing haemodialysis. Furthermore, the systemic inflammatory response index (SIRI), which combines the absolute values of neutrophils, monocytes, and lymphocytes, is a novel inflammatory index and has been widely considered in disease diagnosis and prognosis evaluation in recent years [8-11].

Owing to different types of bacterial infections, the treatment methods commonly varied $[12,13]$. Therefore, finding molecular indicators for the early identification of different types of bacterial infections will help clinicians choose antiinfective treatment options. This study is aimed at exploring the NLR, PLR, CAR, and SIRI values in the early diagnosis and bacterial-type identification of CRBSI in patients undergoing haemodialysis to provide a reference for the diagnosis and treatment of CRBSI.

\section{Patients and Methods}

2.1. Study Patients. From January 2015 to June 2021, a total of 296 patients undergoing haemodialysis using CVCs to establish vascular access in the Blood Purification Centre of Suzhou Hospital Affiliated with Nanjing Medical University were retrospectively selected as study patients. The patients were divided into the infected (58) and uninfected (238) groups based on the CRBSI development.
Patients who met the following criteria were included: (1) the CRBSI diagnosed based on the relevant diagnostic criteria issued by the US Centres for Disease Control in 2008, (2) aged $>18$ years, and (3) Seldinger technique for deep venipuncture catheterisation.

Patients with the following conditions were excluded: (1) skin infection before catheterisation; (2) complications such as serious heart-, brain-, or liver-related diseases; (3) other types of infections; and (4) incomplete data on clinical and laboratory indicators or nosocomial infection aetiology. Among the 296 patients, 160 were men and 136 were women, with ages ranging from 35 to 79 years, with an average age of $48.64 \pm 9.13$ years.

All procedures in this study were following the Declaration of Helsinki. This study was approved by the hospital ethics committee (approval number: KL901167).

2.2. Collection of Infection Status and Clinical Data. By reviewing the medical records of patients and hospital infection monitoring system, the occurrence and distribution characteristics of the pathogens were retrospectively collected. Furthermore, the patients' age, sex, primary disease, catheterisation site, catheter type, catheterisation days, dialysis time, and whether to use immunosuppressive agents were recorded.

2.3. Collection of Laboratory Indicators. By searching the patients' medical records and laboratory forms, indicators including neutrophils (N), lymphocytes (L), platelets (PLT), monocytes (M) counts, C-reactive protein (CRP), and albumin were collected. Then, the NLR, PLR, CAR, and SIRI were calculated. The SIRI was calculated as follows: $N \times M / L$. The laboratory test values within $48 \mathrm{~h}$ before the blood culture and 2 weeks after the anti-infective treatment were recorded in patients with CRBSI, whereas the test values were taken on admission in those without CRBSI.

2.4. Statistical Analysis. The SPSS 22.0 was used for all statistical analyses. Quantitative variables with normal distribution were described as mean and standard deviation (SD) and compared using the $t$-test. Qualitative data were described as frequency and percentage and compared using the chi-square test. The receiver operating characteristic (ROC) curve was used to evaluate the efficacy of inflammatory composite indices in the early diagnosis of CRBSI in patients undergoing haemodialysis. $P<0.05$ was considered statistically significant.

\section{Results}

3.1. Aetiological and General Characteristics. CRBSI occurred in 58 of 296 patients undergoing haemodialysis, with an infection rate of $19.59 \%$. Using the samples of the 58 infected patients, 67 strains of pathogenic bacteria were cultured, including 40 gram-negative bacteria (59.70\%), 23 gram-positive bacteria (34.32\%), and 4 fungi (5.97\%) (Table 1).

Differences in the catheter-type distribution were observed between the infected and uninfected groups, and the proportion of patients with temporary catheters in the 
TABLE 2: The characteristics of patients with and without CRBSI.

\begin{tabular}{|c|c|c|c|c|c|}
\hline Characteristics & $N$ & Infected group $(n=58)$ & Noninfected group $(n=238)$ & $t / \chi^{2}$ & $P$ \\
\hline Age (years) & & $49.12 \pm 9.93$ & $48.53 \pm 8.16$ & 0.833 & 0.402 \\
\hline $\operatorname{Sex}(N, \%)$ & & & & 2.539 & 0.111 \\
\hline Male & 156 & $36(62.07)$ & $120(50.42)$ & & \\
\hline Female & 140 & $22(37.93)$ & $118(49.58)$ & & \\
\hline Protopathy $(N, \%)$ & & & & 0.781 & 0.941 \\
\hline Chronic glomerulonephritis & 76 & $14(24.13)$ & $62(26.05)$ & & \\
\hline Diabetic nephropathy & 85 & $17(29.31)$ & $68(28.57)$ & & \\
\hline Hypertensive nephropathy & 112 & $21(36.21)$ & $91(38.24)$ & & \\
\hline Nephrotic syndrome & 7 & $2(3.45)$ & $5(2.10)$ & & \\
\hline Others & 16 & $4(6.90)$ & $12(5.04)$ & & \\
\hline Cathetering site $(N, \%)$ & & & & 0.108 & 0.743 \\
\hline Jugular vein & 230 & $46(79.31)$ & $184(77.31)$ & & \\
\hline Femoral vein & 66 & $12(20.69)$ & $54(22.69)$ & & \\
\hline Catheter type $(N, \%)$ & & & & 5.466 & 0.019 \\
\hline Long-term catheter & 192 & $30(51.72)$ & $162(68.07)$ & & \\
\hline Temporary catheter & 104 & $28(48.28)$ & $76(31.93)$ & & \\
\hline Cathetering days $(N, \%)$ & & & & 0.212 & 0.645 \\
\hline$<28$ days & 181 & $37(63.79)$ & $144(60.50)$ & & \\
\hline$\geq 28$ days & 115 & $21(36.21)$ & $94(39.50)$ & & \\
\hline Dialysis time $(N, \%)$ & & & & 3.744 & 0.053 \\
\hline$<1$ years & 223 & $38(65.52)$ & $185(77.74)$ & & \\
\hline$\geq 1$ years & 73 & $20(34.48)$ & $53(22.26)$ & & \\
\hline Whether use immunosuppressants $(N, \%)$ & & & & 0.022 & 0.883 \\
\hline No & 269 & $53(91.38)$ & $216(90.76)$ & & \\
\hline Yes & 27 & $5(8.62)$ & $22(9.24)$ & & \\
\hline
\end{tabular}

infected group was significantly higher than that in the uninfected group $(P<0.05)$, although no significant differences were found in age, sex, protopathy, catheterisation site, catheterisation days, dialysis time, and use of immunosuppressive agents between the two groups $(P>0.05)$. The results are demonstrated in Table 2.

3.2. Comparison of NLR, PLR, CAR, and SIRI between Patients with and without CRBSI. The NLR, PLR, CAR, and SIRI values in the infected group were $4.77 \pm 0.65$, $169.50 \pm 26.70,1.04 \pm 0.17$, and $4.06 \pm 0.56$, respectively, which were significantly higher than those in the uninfected group (NLR, PLR, CAR, and SIRI were $3.93 \pm 0.51,158.14$ $\pm 21.90,0.80 \pm 0.15$, and $2.86 \pm 0.42$, respectively, all $P<$ 0.05) (Figure 1).

3.3. Comparison of NLR, PLR, CAR, and SIRI in Patients with CRBSI before and after Anti-Infective Treatment. After an anti-infective treatment, the NLR, PLR, CAR, and SIRI values in patients with CRBSI were $3.75 \pm 0.31,152.32 \pm$ $22.13,0.76 \pm 0.12$, and $2.78 \pm 0.31$, respectively, which were significantly lower than those at pretreatment (all $P<0.001$ ) (Figure 2).

3.4. ROC Curve Analysis of NLR, PLR, CAR, and SIRI for the Early Diagnosis of CRBSI. To determine the availability of
NLR, PLR, CAR, and SIRI for the early diagnosis of CRBSI, ROC curve analysis, a broadly appreciated objective statistical method, was performed. Whether CRBSI developed in patients undergoing haemodialysis was taken as the variable state $($ no $=0$, yes $=1$ ), and NLR, PLR, CAR, and SIRI were considered as test variables, respectively. Finally, except the PLR, the NLR, CAR, and SIRI were effective diagnostic markers in patients with CRBSI with cut-off values of 4.485 (area under the curve $(\mathrm{AUC})=0.827,95 \% \mathrm{CI}=0.768$ -0.887 , sensitivity $=70.69 \%$, and specificity $=83.61 \%$ ), $0.975(\mathrm{AUC}=0.836,95 \% \mathrm{CI}=0.779-0.892$, sensitivity $=$ $65.51 \%$, and specificity $=87.39 \%)$, and $3.390(\mathrm{AUC}=0.947$, $95 \% \mathrm{CI}=0.919-0.976$, sensitivity $=87.93 \%$, and specificity $=86.56 \%)$ (Figure 3(a)).

3.5. NLR, PLR, CAR, and SIRI Values in Differentiating Patients with Blood-Cultured Gram-Negative $\left(G^{-}\right)$and Gram-Positive $\left(G^{+}\right)$Bacteria. The blood culture results indicated that 47 of 58 patients with CRBSI were single bacterial infections, including $28 \mathrm{G}^{-}$bacterial infections and $19 \mathrm{G}^{+}$ bacterial infections. The NLR, PLR, CAR, and SIRI values in the $\mathrm{G}^{-}$group were $4.72 \pm 0.62,168.71 \pm 15.24,1.08 \pm$ 0.12 , and $4.23 \pm 0.52$, respectively, and those in the G+ group were $4.48 \pm 0.59,165.42 \pm 14.86,0.95 \pm 0.08$, and $3.81 \pm 0.44$, respectively. Comparison results between the two groups showed that CAR and SIRI values in the $\mathrm{G}^{-}$ 


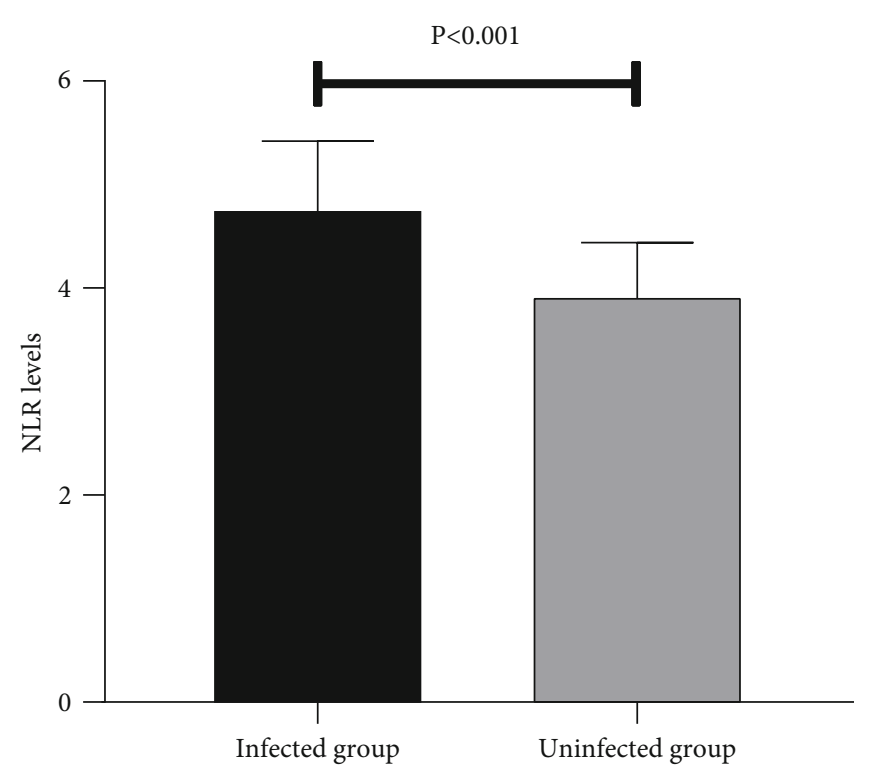

(a)

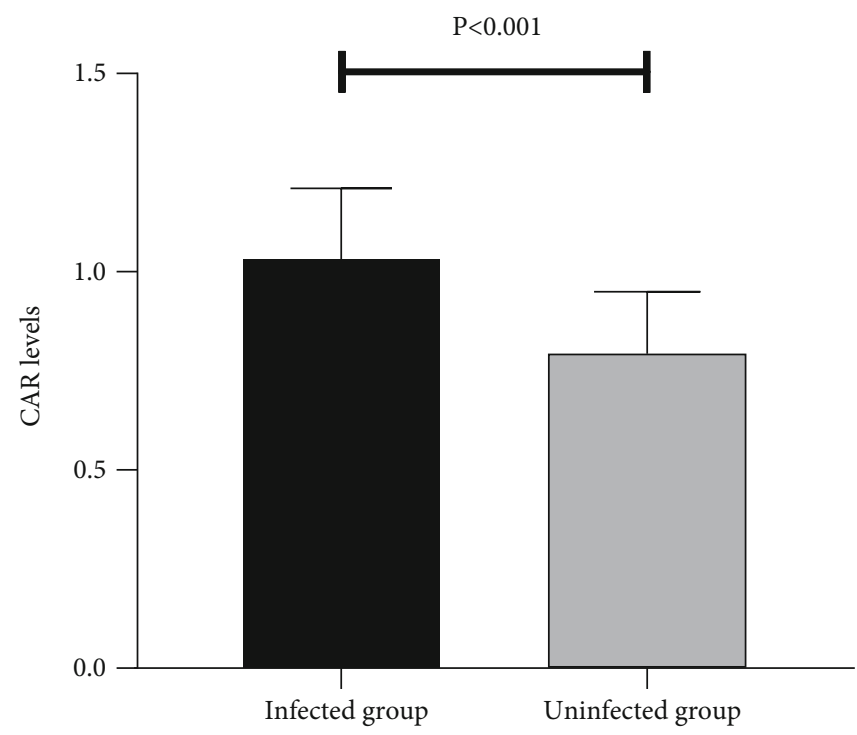

(c)

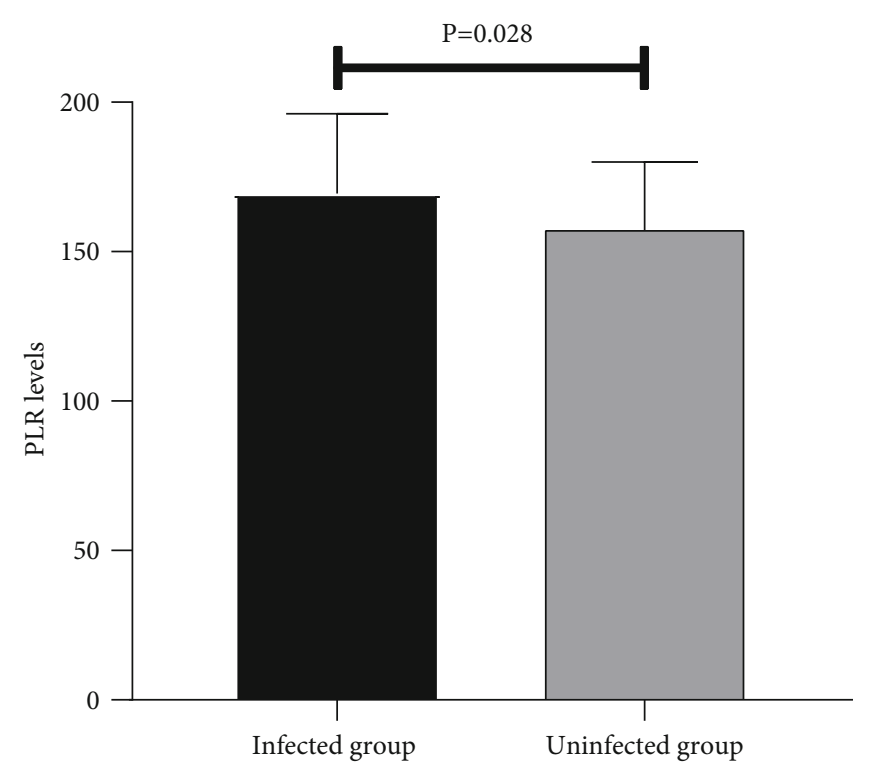

(b)

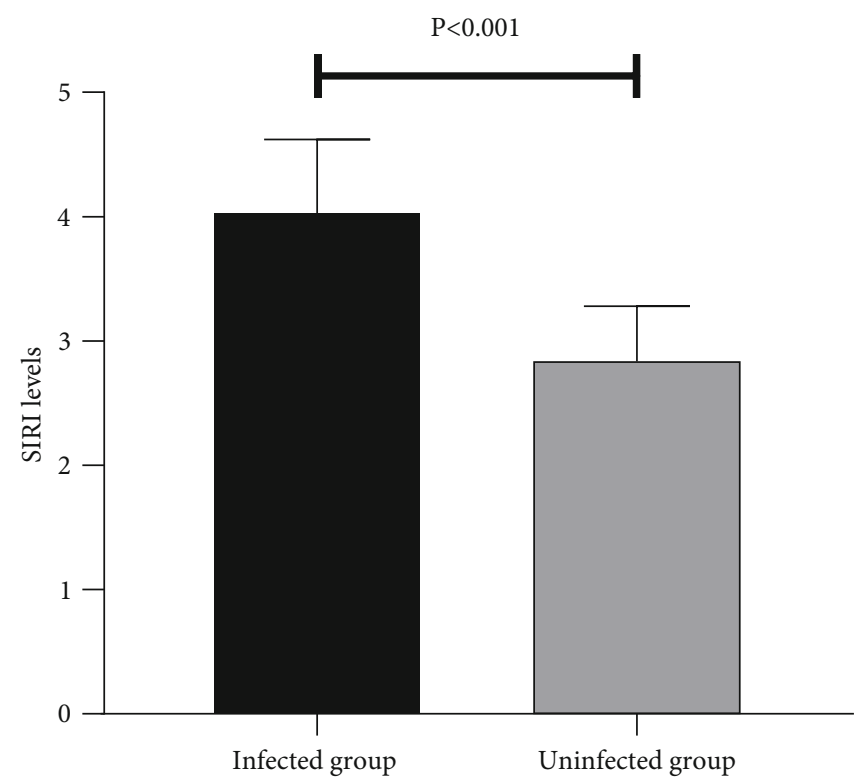

(d)

FIGURE 1: Comparison of inflammatory index values between the infected and uninfected groups: (a) comparison of NLR values; (b) comparison of PLR values; (c) comparison of CAR values; (d) comparison of SIRI values. NLR: neutrophil-to-lymphocyte ratio; PLR: platelet-to-lymphocyte ratio; CAR: C-reactive protein-to-albumin ratio; SIRI: systemic inflammatory response index.

group were significantly higher than those in the $\mathrm{G}^{+}$group $(P<0.05)$, whereas NLR and PLR values showed no statistical significance between the two groups $(P>0.05)$ (Figure 4).

ROC curve analysis was performed to evaluate whether CAR or SIRI could be used to identify different bacterial types. The bacterial type was considered as the state variable $\left(\mathrm{G}^{+}=0\right.$, $\mathrm{G}^{-}=1$ ), and CAR and SIRI were regarded as test variables. CAR and SIRI showed diagnostic values in $\mathrm{G}^{-}$bacterial infection with cut-off values of 0.775 (AUC $=0.693,95 \% \mathrm{CI}=$ $0.537-0.848$, sensitivity $=50.00 \%$, and specificity $=84.21 \%$ ) and 2.585 ( $\mathrm{AUC}=0.821,95 \% \mathrm{CI}=0.700-0.942$, sensitivity $=75.00 \%$, and specificity $=78.95 \%)($ Figure $3(\mathrm{~b})$ ).

\section{Discussion}

CRBSI is the most clinically relevant infection owing to the potential progression to sepsis and death [14]. If the treatment for CRBSI is untimely, it will enormously endanger the health of patients and increase the economic burden on their families [15]. Blood culture is currently the gold standard to diagnose bloodstream infection. However, due to the long culture cycle and low positive rate of blood culture, patients may miss the optimal opportunity for the treatment if relying on blood culture alone [16]. Therefore, a rapid and simple indicator is urgently needed for the early diagnosis of bloodstream infection. This study discussed the 


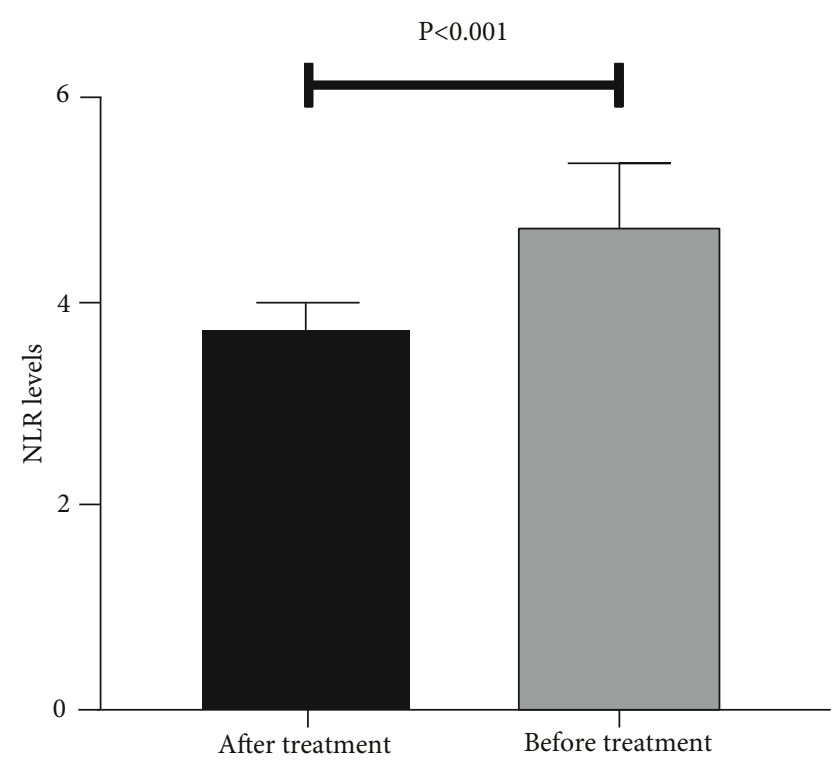

(a)

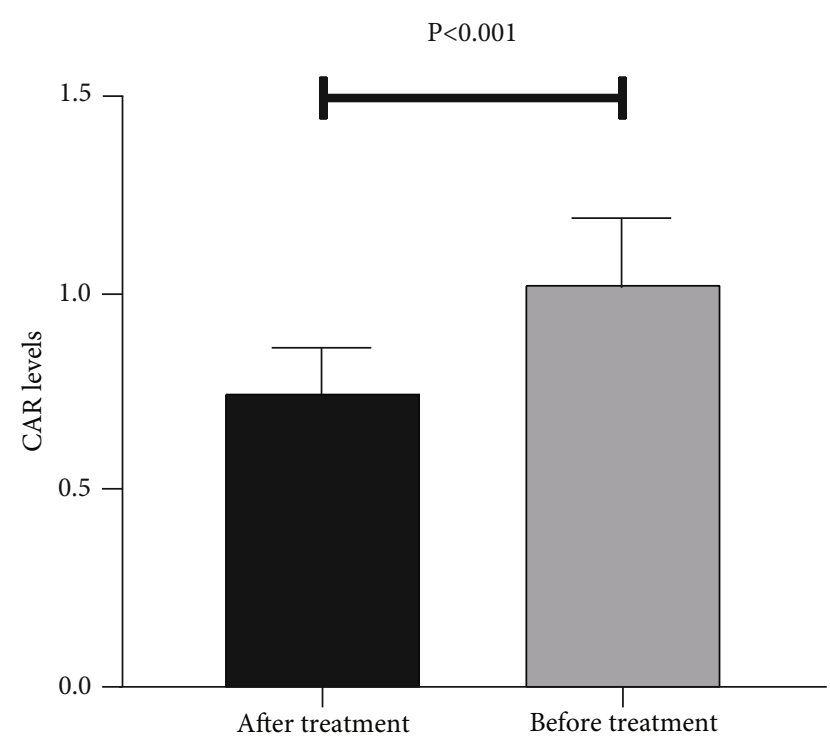

(c)

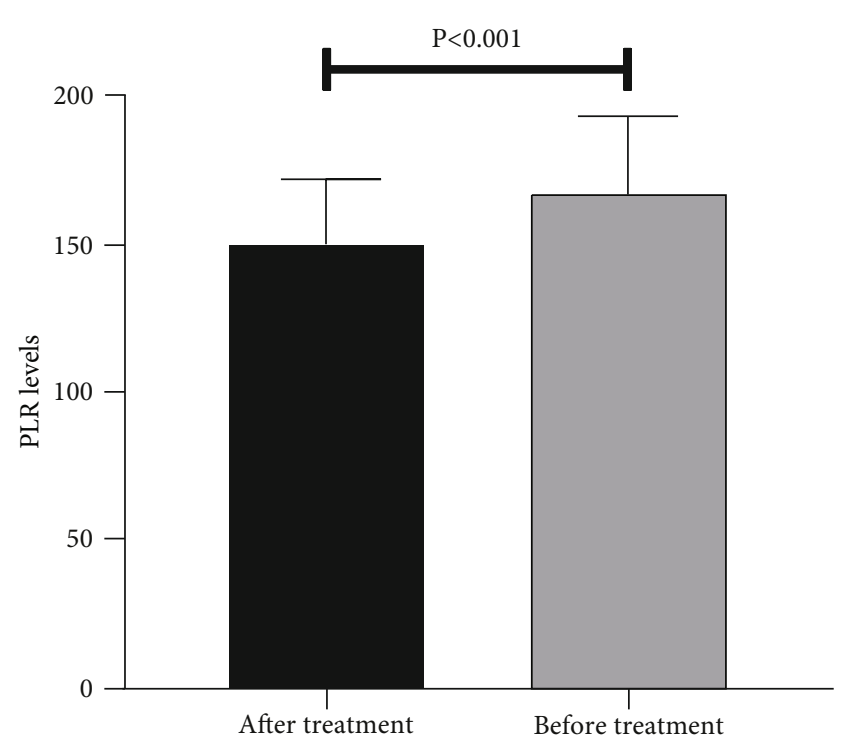

(b)

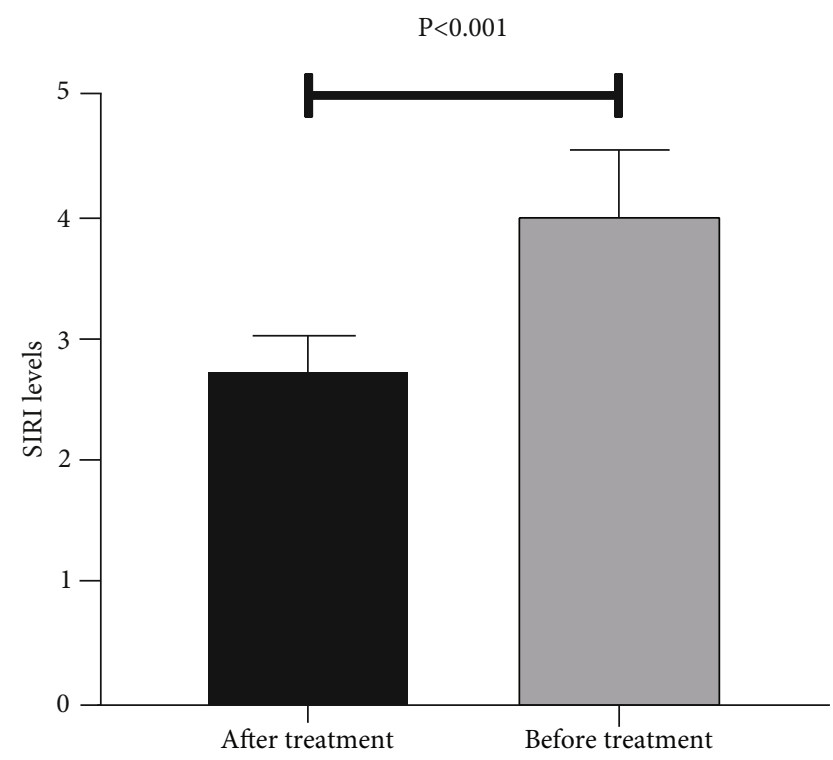

(d)

Figure 2: Comparison of NLR, PLR, CAR, and SIRI values before and after anti-infective treatment: (a) comparison of NLR values; (b) comparison of PLR values; (c) comparison of CAR values; (d) comparison of SIRI values. NLR: neutrophil-to-lymphocyte ratio; PLR: platelet-to-lymphocyte ratio; CAR: C-reactive protein-to-albumin ratio; SIRI: systemic inflammatory response index.

application of NLR, PLR, CAR, and SIRI in the early diagnosis of patients with CRBSI.

Previous studies have shown that the incidence of CRBSI in patients undergoing maintenance haemodialysis is approximately $24 \%$ [17]. The study results showed that the incidence of CRBSI in patients undergoing haemodialysis was $19.59 \%$, which was slightly lower than that reported by Fram et al. [18]. Gram-negative bacteria were the main pathogenic bacteria (59.7\%), followed by gram-positive bacteria $(34.3 \%)$ and fungi accounting for the lowest proportion (6.0\%). The top five pathogenic bacteria were Escherichia coli (47.5\%), Staphylococcus aureus (39.1\%), Pseudomonas aeruginosa (25.0\%), Staphylococcus cephalus (21.7\%), and
Enterococcus faecium (17.4\%), all of which were common bacteria on the skin surface and widely occurred on the body surface of the medical staff and patients. In invasive diagnostic and therapeutic procedures, pathogenic bacteria on the skin surface of medical staff and patients are the main source of pathogenic bacteria for CRBSI. Moreover, the immune system of patients undergoing haemodialysis is compromised. Hence, they are predisposed to infection through the catheter route while undergoing treatment or dialysis. Therefore, to reduce infections caused by pathogenic bacteria, standard handwashing and strict adherence to aseptic practices are essential to prevent bacteria from passing from the skin to the bloodstream. 

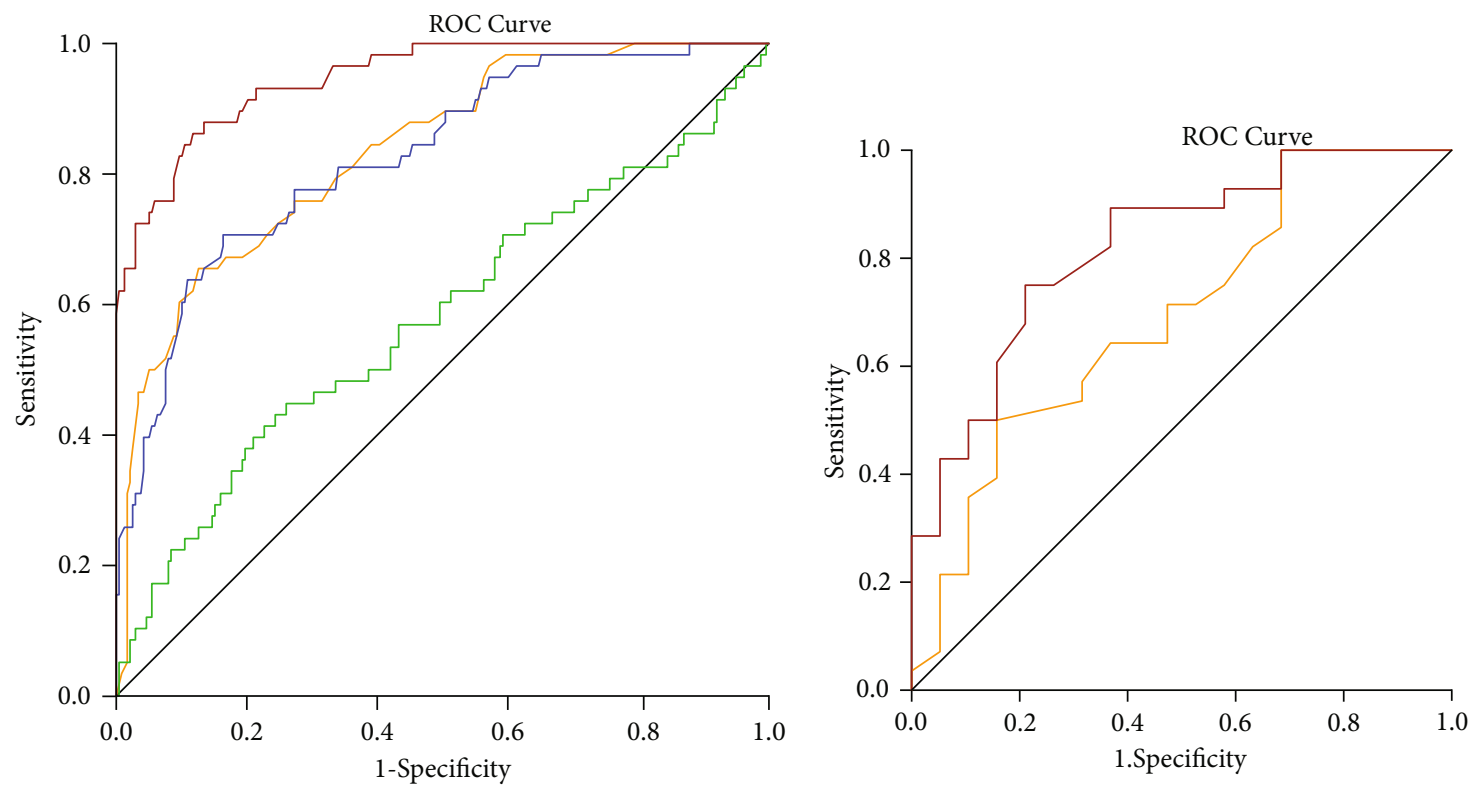

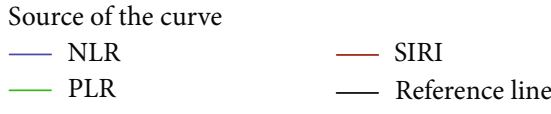

(a)

$$
\begin{aligned}
& \text { Source of the curve } \\
& \text { CAR } \\
& \text { SIRI }
\end{aligned}
$$

(b)

FIGURE 3: ROC curve of the inflammatory composite index for diagnosing CRBSI and identifying different bacterial types: (a) ROC curves for NLR, PLR, CAR, and SIRI to diagnose CRBSI; (b) ROC curves for CAR and SIRI to distinguish gram-negative and gram-positive bacterial infections. ROC: receiver operator characteristic; NLR: neutrophil-to-lymphocyte ratio; PLR: platelet-to-lymphocyte ratio; CAR: C-reactive protein-to-albumin ratio; SIRI: systemic inflammatory response index; CRBSI: catheter-related bloodstream infection.

Our study found a significant difference in the distribution of catheter types between patients undergoing haemodialysis with and without CRBSI. The proportion of patients with nontunnelled temporary haemodialysis catheters in the CRBSI group was significantly higher than that in the non-CRBSI group, which may be related to the fact that the temporary catheter more likely slips and without the tunnel to prevent bacterial invasion [19]. Inflammatory indicators in patients undergoing haemodialysis were retrospectively collected and found that NLR, PLR, CAR, and SIRI were all significantly increased in patients with CRBSI than those without. Furthermore, the results suggested that NLR, PLR, CAR, and SIRI values after an anti-infective treatment were significantly lower than at pretreatment. Therefore, we assumed that these indicators may be applied in the early diagnosis of CRBSI to a certain degree.

Results of ROC analysis demonstrated that SIRI has decent diagnostic efficiency, with sensitivity and specificity of $87.9 \%$ and $86.6 \%$, respectively. Nevertheless, the application of NLR and CAR in the diagnosis of CRBSI has some limitations because of moderate sensitivity or specificity. Therefore, applying SIRI for the early diagnosis of CRBSI is conducive to the timely targeted treatment, which can improve the prognosis of patients. Wu et al. [5] found that NLR had a certain early diagnostic value for bloodstream infection in patients in the emergency department, but its diagnostic sensitivity and specificity were not high. In this study, NLR had good specificity (83.6\%) to diagnose CRBSI, whereas its sensitivity was only $70.7 \%$. Hence, using NLR alone to diagnose CRBSI may increase the rate of misdiagnosis.

Fang et al. [20] showed that compared with patients undergoing peritoneal dialysis without infection, NLR and PLR values in patients with infections were significantly higher. Additionally, although the efficacy of NLR and PLR alone in diagnosing peritoneal dialysis-associated infection was not that good, the diagnostic sensitivity and specificity of NLR combined with PLR were pleasing and better than that of high-sensitivity C-reactive protein. Yigit et al. [21] showed that CAR had a high predictive value in periprosthetic infection after total joint arthroplasty; however, the results of our study indicated that the CAR was not satisfactory for the early diagnosis of CRBSI, which may be because of the different types of infection. SIRI is a new compound inflammatory biomarker based on traditional inflammatory cells, including $\mathrm{N}, \mathrm{L}$, and $\mathrm{M}$, which can comprehensively reflect the inflammatory state of the body.

Recent studies have demonstrated the diagnostic value of some other markers of bloodstream infection. Shokripour and Omidifar [22] reported that serum procalcitonin, but not CRP, was statistically significant in distinguishing bloodstream infections in patients with malignancy. However, the sensitivity and specificity were approximately $61.52 \%$ and $80.08 \%$; hence, the diagnostic efficiency of serum procalcitonin in diagnosing bloodstream infections is mediocre. Chen et al. [23] demonstrated that CRP and serum procalcitonin 


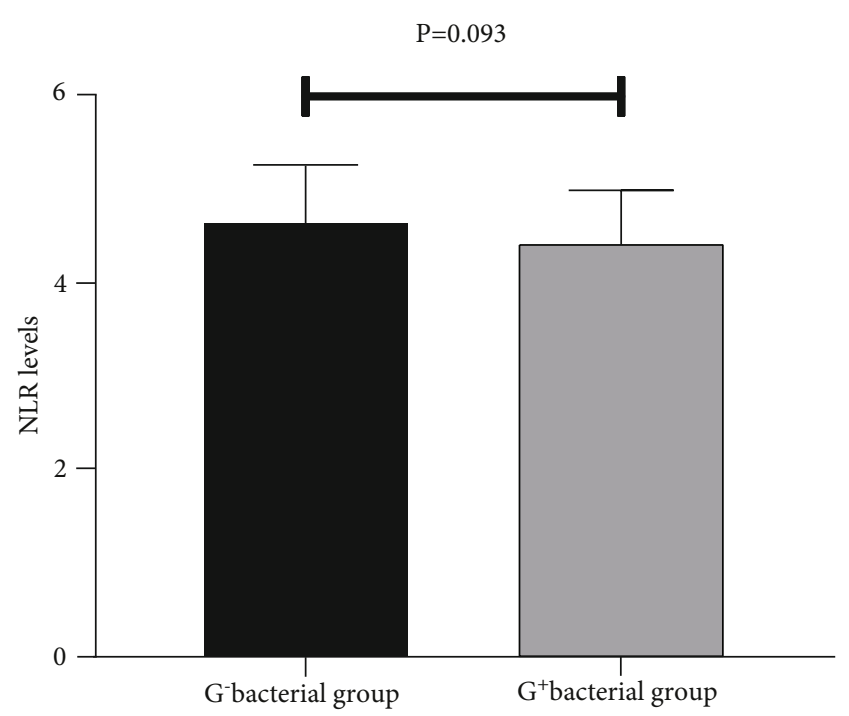

(a)

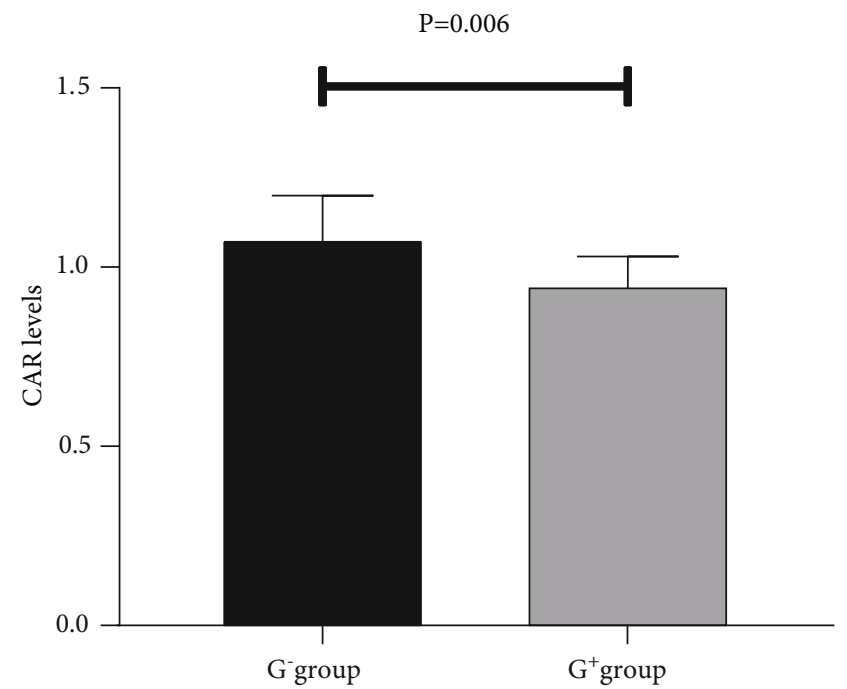

(c)

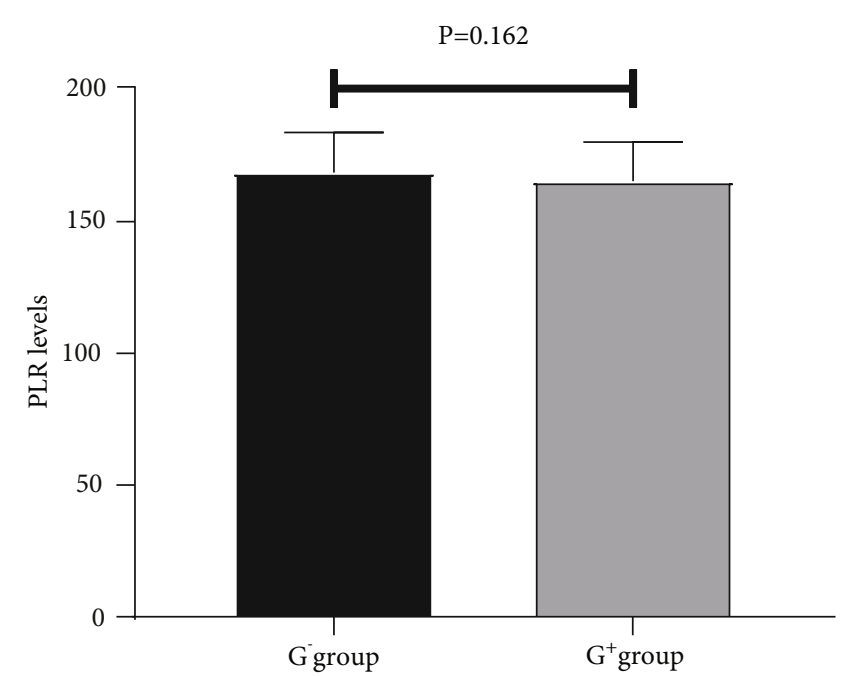

(b)

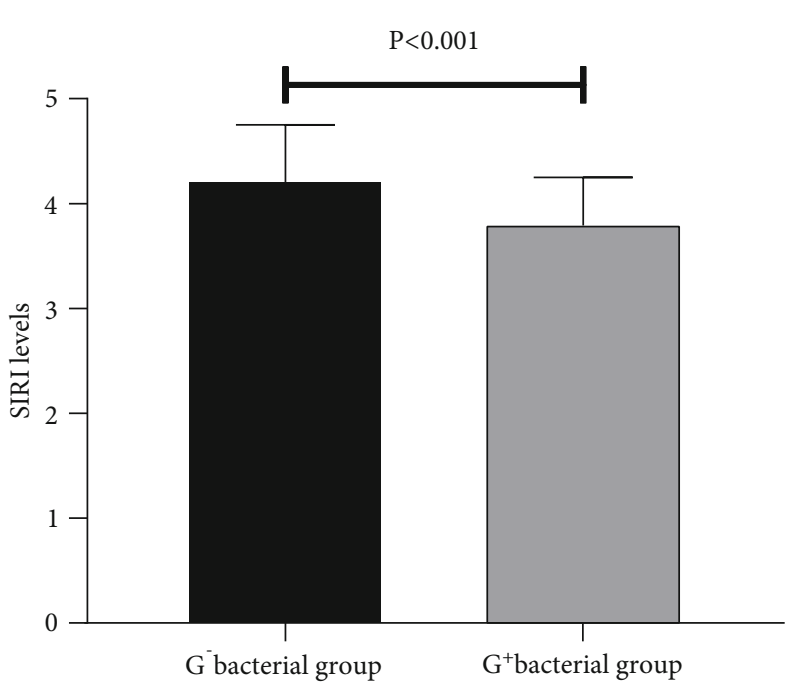

(d)

FIGURE 4: Comparison of NLR, PLR, CAR, and SIRI values between gram-negative and gram-positive groups: (a) comparison of NLR values; (b) comparison of PLR values; (c) comparison of CAR values; (d) comparison of SIRI values. NLR: neutrophil-to-lymphocyte ratio; PLR: platelet-to-lymphocyte ratio; CAR: C-reactive protein-to-albumin ratio; SIRI: systemic inflammatory response index.

were effective for the early diagnosis of $\mathrm{G}^{-}$bacterial bloodstream infection with a sensitivity of $71.4 \%$ and $64.3 \%$ and specificity of $96.2 \%$ and $80.8 \%$, respectively. Although the specificities were outstanding, the sensitivities were ordinary. In our study, SIRI was found to have favourable diagnostic efficiency, with sensitivity and specificity of $87.9 \%$ and $86.6 \%$, respectively. Therefore, SIRI was more accurate than previously reported markers for bloodstream infections.

Furthermore, owing to its convenience and economy, SIRI plays an important role in predicting the occurrence, development, and prognosis of various diseases. Topkan et al. [14] have found that SIRI could be a novel, sound, and independent predictor of survival outcomes in patients with newly diagnosed glioblastoma multiforme who underwent postoperative Stupp protocol. Chen et al. [24] have demonstrated that SIRI was an effective inflammatory marker to estimate hyperuricaemia in rural Chinese women and was likely to optimise the risk stratification and prevention of hyperuricaemia. A recent study [25] has shown that SIRI was significantly higher in patients with coronavirus disease 2019 (COVID-19) than those in the control group, whereas its diagnostic value in COVID-19 was moderate. In this study, SIRI was used for the early diagnosis of CRBSI in patients undergoing haemodialysis for the first time, and the diagnostic effect was increasing. Therefore, SIRI could be used as a novel indicator for the early diagnosis of CRBSI in patients undergoing haemodialysis.

Furthermore, the results indicated that CAR and SIRI values in the $\mathrm{G}^{-}$bacteria were significantly higher than those in the $\mathrm{G}^{+}$bacteria, whereas NLR and PLR values were not significantly different between the two groups, indicating that CAR and SIRI may play a role in identifying the types 
of bacterial infections. Further ROC curve analysis displayed that although CAR and SIRI are statistically significant in differentiating between $\mathrm{G}^{-}$and $\mathrm{G}^{+}$bacterial infections, the effectiveness in practical application is mediocre. Sumardi et al. [26] have found that NLR in $\mathrm{G}^{-}$bacteria was lower than that in $\mathrm{G}^{+}$bacteria in patients with sepsis. A recent study [27] has shown that the sensitivity and specificity of NLR in differentiating bloodstream infections caused by $\mathrm{G}^{-}$ and $\mathrm{G}^{+}$bacteria were $73.70 \%$ and $53.10 \%$, respectively. However, certain limitations exist in recognising different types of bacteria using these composite inflammatory indicators. In the future, further studies on valuable molecular markers for the early identification of different types of bacterial infections are needed.

The current study demonstrated several potential weaknesses. First, all patients included in this study were from the same hospital; therefore, selection bias was inevitable. Second, this study found that the type of catheter was also an influencing factor in the CRBSI development in patients undergoing haemodialysis. However, subgroup analyses were not performed for different types of catheters. Finally, we did not dynamically monitor the NLR, PLR, CAR, and SIRI values of patients with CRBSI pre-, during, and postinfection. In future studies, these aspects will be evaluated when manpower and material resources allow.

\section{Conclusion}

The outcomes of our current retrospective study indicated that NLR, PLR, CAR, and SIRI were higher in patients with CRBSI than those without. SIRI can be used as a novel indicator for the early diagnosis of CRBSI in patients undergoing haemodialysis.

\section{Abbreviations}

CVC: Central venous catheter

CRBSI: Catheterism-related bloodstream infection

NLR: Neutrophil-to-lymphocyte ratio

PLR: Platelet-to-lymphocyte ratio

CAR: C-reactive protein-to-albumin ratio

SIRI: Inflammatory response index

N: $\quad$ Neutrophils

L: $\quad$ Lymphocytes

PLT: $\quad$ Platelets

M: $\quad$ Monocytes

CRP: C-reactive protein

SD: $\quad$ Standard deviation

ROC: Receiver operating characteristic

AUC: Area under the curve

G-: Gram-negative

$\mathrm{G}+$ : $\quad$ Gram-positive.

\section{Data Availability}

The datasets used and/or analyzed during the present study are available from the corresponding author on reasonable request.

\section{Conflicts of Interest}

The authors declare no competing interests.

\section{Authors' Contributions}

YW is the guarantor of this work; JY, HW, and QH performed data collection; JY performed statistical analysis and manuscript writing; JW performed critical revision of the manuscript.

\section{Acknowledgments}

We thank all patients and blood donors who participated in our study. This study was funded by grants from the Suzhou Science and Technology Development Plan Project (SYSD2020144) and the Science and Technology Development Project of Nanjing Medical University (NMUB2020259).

\section{References}

[1] C. F. Chen and C. C. Lin, "The association of genotype polymorphisms with vascular access patency in hemodialysis patients," The Journal of Vascular Access, vol. 20, pp. 24-30, 2019.

[2] L. Golestaneh and M. H. Mokrzycki, "Prevention of hemodialysis catheter infections: ointments, dressings, locks, and catheter hub devices," Hemodialysis International, vol. 22, pp. S75s82, 2018.

[3] L. Kumbar and J. Yee, "Current concepts in hemodialysis vascular access infections," Advances in Chronic Kidney Disease, vol. 26, pp. 16-22, 2019.

[4] E. Serna, A. Parra-Llorca, J. Panadero, M. Vento, and M. Cernada, "miRNomic signature in very low birth-weight neonates discriminates late-onset gram-positive sepsis from controls," Diagnostics, vol. 11, no. 8, p. 1389, 2021.

[5] J. Wu, X. Zhang, H. Liu, N. Guo, Q. Pan, and Y. Wang, "RDW, NLR and RLR in predicting liver failure and prognosis in patients with hepatitis E virus infection," Clinical Biochemistry, vol. 63, pp. 24-31, 2019.

[6] G. Zhao, J. Chen, J. Wang et al., "Predictive values of the postoperative neutrophil-to-lymphocyte ratio, platelet-tolymphocyte ratio, and lymphocyte-to-monocyte ratio for the diagnosis of early periprosthetic joint infections: a preliminary study," Journal of Orthopaedic Surgery and Research, vol. 15, p. $571,2020$.

[7] C. S. Hong and S. P. Ryan, "Predicting success of two-stage exchange for prosthetic joint infection using C-reactive protein/albumin ratio," Advances in Orthopedics, vol. 2019, Article ID 6521941, 2019.

[8] J. Ni, K. Wang, H. Zhang et al., "Prognostic value of the systemic inflammatory response index in patients undergoing radical cystectomy for bladder cancer: a population-based study," Frontiers in Oncology, vol. 11, article 722151, 2021.

[9] J. Wu, N. Guo, X. Zhang et al., "HEV-LFS: a novel scoring model for patients with hepatitis E virus-related liver failure," Journal of Viral Hepatitis, vol. 26, no. 11, pp. 1334-1343, 2019.

[10] S. Yun, H. J. Yi, D. H. Lee, and J. H. Sung, "Systemic inflammation response index and systemic immune-inflammation index for predicting the prognosis of patients with aneurysmal 
subarachnoid hemorrhage," Journal of Stroke and Cerebrovascular Diseases, vol. 30, no. 8, article 105861, 2021.

[11] G.-h. Zhai, W. Zhang, Z. Xiang et al., "Diagnostic value of sIL$2 \mathrm{R}, \mathrm{TNF}-\alpha$ and PCT for sepsis infection in patients with closed abdominal injury complicated with severe multiple abdominal injuries," Frontiers in Immunology, vol. 12, article 741268, 2021.

[12] C. Y. Yang, C. H. Lee, C. C. Hsieh, M. Y. Hong, M. J. Chen, and C. C. Lee, "Differential effects of inappropriate empirical antibiotic therapy in adults with community-onset gram-positive and gram-negative aerobe bacteremia," Journal of Infection and Chemotherapy, vol. 26, pp. 222-229, 2020.

[13] M. J. Dowzicky and E. Chmelařová, "Antimicrobial susceptibility of gram-negative and gram-positive bacteria collected from Eastern Europe: results from the tigecycline evaluation and surveillance trial (T.E.S.T.), 2011-2016," Journal of Global Antimicrobial Resistance, vol. 17, pp. 44-52, 2019.

[14] E. Topkan, A. Kucuk, Y. Ozdemir et al., "Systemic inflammation response index predicts survival outcomes in glioblastoma multiforme patients treated with standard Stupp protocol," vol. 2020, Article ID 8628540, pp. 1-10, 2020.

[15] M. Suzuki, N. Satoh, M. Nakamura, S. Horita, G. Seki, and K. Moriya, "Bacteremia in hemodialysis patients," World Journal of Nephrology, vol. 5, pp. 489-496, 2016.

[16] F. Quittnat Pelletier, M. Joarder, S. M. Poutanen, and C. E. Lok, "Evaluating approaches for the diagnosis of hemodialysis catheter-related bloodstream infections," Clinical Journal of the American Society of Nephrology, vol. 11, pp. 847-854, 2016.

[17] G. Loomba, M. Dhandapani, S. Kaur et al., "The effectiveness of personal hygiene practices on non-cuffed central vein catheter-related infection in patients undergoing hemodialysis: a randomized controlled trial," Indian Journal of Nephrology, vol. 29, pp. 267-271, 2019.

[18] D. Fram, M. F. Okuno, M. Taminato et al., "Risk factors for bloodstream infection in patients at a Brazilian hemodialysis center: a case-control study," BMC Infectious Diseases, vol. 15, no. 1, p. 158, 2015.

[19] H. Van Der Meersch, D. De Bacquer, S. J. Vandecasteele et al., "Hemodialysis catheter design and catheter performance: a randomized controlled trial," American Journal of Kidney Diseases, vol. 64, pp. 902-908, 2014.

[20] W. Fang, G. Liu, Q. Li, W. Zhao, X. Chen, and D. Li, "Diagnostic value of NLR and PLR in peritoneal dialysis-associated infection," Journal of Clinical Nephrology, vol. 20, pp. 12-18, 2020.

[21] Ş. Yigit, M. S. Akar, M. A. Şahin, and H. Arslan, "Periprosthetic infection risks and predictive value of C-reactive protein/albumin ratio for total joint arthroplasty," Acta BioMedica, vol. 92, article e2021324, 2021.

[22] M. Shokripour, N. Omidifar, K. Salami, M. Moghadami, and B. Samizadeh, "Diagnostic accuracy of immunologic biomarkers for accurate diagnosis of bloodstream infection in patients with malignancy: procalcitonin in comparison with C-reactive protein," vol. 2020, Article ID 8362109, pp. 1-6, 2020.

[23] W. Chen, L. Zhao, S. Niu et al., "The diagnostic value of different pro-inflammatory factor in early diagnosis of sepsis in patients with bloodstream infection," Zhonghua Wei Zhong Bing Ji Jiu Yi Xue, vol. 26, pp. 165-170, 2014.

[24] M. A.-O. Chen, H. A.-O. X. Wang, W. A.-O. Shi, and Y. A.-O. Sun, "Estimate of prevalent hyperuricemia by systemic inflammation response index: results from a rural Chinese populationvol. 133, no. 2, pp. 242-249.
[25] M. A.-O. Eissa, S. Shaarawy, and M. A.-O. Abdellateif, "The role of different inflammatory indices in the diagnosis of COVID-19," International Journal of General Medicine, vol. Volume 14, pp. 7843-7853, 2021.

[26] U. A.-O. Sumardi, D. A.-O. Prihardianti, and P. A.-O. X. Sudjana, "Is neutrophil-lymphocyte count ratio a better indicator of sepsis with gram-positive bacterial infection?," Indian Journal of Critical Care Medicine, vol. 25, no. 7, pp. 795-799, 2021.

[27] X. Zou, L. Ling, X. Liu, Z. Li, and X. Lu, "Diagnostic value of NLR and SII in different types of bloodstream infection," Laboratory Medicine and Clinical Practice, vol. 18, pp. 2501-2503, 2021. 